\title{
DISCUSSION (Severny and Lyubimkov)
}

SADAKANE: I have six high dispersion IUE spectra of 73 Dra taken in 1978 and 1979. My analysis of the 2556 \& region does not confirm the U II line at 2556.19 A. Also, two other lines of U II at 2562.94 \& and 2565.41 A can not be confirmed in 73 Dra. The same thing occurs in the 1979 spectrum of HR $465=\mathrm{HD}$ 9996, in which strong U II lines close to 3859 A are confirmed. Possibly, the U II lines in 73 Dra are strongly variable.

SEVERNY: Yes. [Inaudible] summed spectra from IUE and also from Earth for about the same phase. They did not find such a discrepancy with our result, they found traces of this line quite surely. It may be that the abundance depends on the phase because it is a variable star.

ROMANOV: Have you an interpretation of the variation of the abundance of uranium for 73 Dra?

SEVERNY: I prefer to avoid any conclusions or interpretations, because the investigation of the whole set of stars for heavy element abundances is not complete.

ADELMAN: I have looked at 21 Aquilae both in the optical and the ultraviolet. It is not a peculiar A star, but it is a rather sharplined slightly reddened B7 or B8 IV or $V$ star.

SEVERNY: Ultraviolet spectra of this star are not yet analysed by us. COWLEY: In HR 465, we found that we could interpret the line at 3859 A as chromium at phases when the star was Cr-strong. There are $\mathrm{Cr}$-weak phases in that star, and we [Cowley, Aikman \& Fisher, Publs. DAO, 15, p37, 1977] believe in the uranium at those phases. In 73 Dra, the 3859 A line can be very strong, but that star also has very strong $\mathrm{Cr}$. Resolution of this question depends on having atomic data in the ultraviolet and more resolution, perhaps on the Hubble Space Telescope. Overabundance factors of 100 to 1000 are not unexpected, in view of the overabundances of the lanthanide rare earths. It's one thing to say that the actinides could also be overabundant by those factors, but quite a different thing to prove it! I'm not sure that we have sufficiently good data yet to make a definite conclusion.

SEVERNY: We have also looked at your published line profile, and it agrees quite well with the spectra taken at the Bulgarian national observatory. 